\title{
nature
}

\section{A stain on Italian reforms}

Italy's principal funding agency has missed an opportunity to enhance the prestige of its institutes. In appointing its first crop of new directors, it has conspicuously avoided some candidates of the highest calibre.

\begin{abstract}
A year ago, it seemed as if Italy's stodgy National Research Council (CNR) was taking its reform programme seriously. It had drawn up plans to merge its 330 institutes and centres to 100 or so larger units of 'critical mass'. And, with great fanfare, it announced in December its intentions of recruiting directors to the new institutes from the international community - a radical change from its traditional tendency to recruit from its own ranks. Moreover, advertisements referred to the need for 'continual, original and sound' research experience. The CNR claimed that the new research directors were key to ensuring that the reforms delivered in terms of high-quality science.

Disappointingly, the first round of appointments, for the largest of the new institutes, suggests business as usual. Of the first 22 appointments selected last month, all are Italian scientists who have served as CNR directors. And there are examples of clear injustices, where scientists with significant scientific and research-management experience have been passed over in favour of those of lesser merit. In one case, a leading scientist was passed over in favour of another with one-tenth of his publication output in the past decade. Moreover, the average citation rate of the rejected candidate was three times that of the winner. There is no mention of what factors led to the choice.

What went wrong with the plan for the CNR to break out of its mould? One key issue is that it was not given a budget for its reform essential for making the positions attractive to top researchers from other countries. Not surprisingly, few highly qualified foreigners applied. But why there were no appointments of Italian scientists from
\end{abstract}

outside the CNR deserves explanation, as do those aberrant choices.

Another problem is the way in which the selection process took place. The procedures were out of line with international norms. CNR president Lucio Bianco is responsible for the final decision, but he is advised by (and heads) his Consiglio Direttivo, a committee of eight academics from all disciplines, from medicine to law. These committee members vote on candidates for each new institute directorship.

The Consiglio Direttivo did turn to experts for help. It appointed special commissions for each directorship, comprising three experts in the relevant field. But very few outsiders were brought in to ensure truly independent advice. The first 21 commissions whose names are publicly available included only four foreigners.

Moreover, the consiglio asked the commissions to provide unranked short-lists for its less technically expert members to choose from. And non-unanimous cases were combined to be voted on as a group rather than case by case. This system leaves room for suspicion of vote exchange - 'vote for my candidate and I'll owe you a favour' the bad habit that the reforms should have forced the CNR to lose.

If the CNR appointments continue along the lines of these first ones, then it needs to come up publicly with a plausible explanation for why it cannot attract, or appoint, to key positions scientists from beyond its own ranks. Under attack from the new centre-right government of Silvio Berlusconi for inefficiency, the CNR needs more than ever to prove that it stands for research excellence and can strive to achieve it by seeing beyond its own highly politicized horizons.

\section{Visionary experimental designs}

\section{A collaboration marrying epidemiology and genomics should provide a much-needed boost to analytical rigour.}

$\mathrm{T}$ he widespread astonishment following the unconfirmed allegation by the UK government that resear chers spent five years accidentally testing cattle instead of sheep brains for BSE is encouraging. It shows that politicians and the public alike appreciate a basic tenet of experimental design: be sure of the identity of what you are studying.

It may be shocking to some, therefore, that biologists knowingly transgress this rule daily. Genome databases are polluted with incorrect gene functions that are mistakenly assigned through researchers' uncritical faith in the results of BLAST algorithms. Powerful 'blackbox' software packages mean researchers may plug data in, and get results out, with little thought for the rationale and caveats of the inbetween. Many reported associations between diseases and DNA variants specific to particular regions of the genome have also recently emerged as being spurious or irreproducible (see Nature Rev. Genet. 2, 91-99; 2001). Here the explanation often seems to lie in researchers' unfamiliarity with the rigour needed in the statistical and experimental design of such population experiments.

The epitome of the required rigour is perhaps exemplified by the field of epidemiology, steeped in statistics and experimental design. Classical epidemiology has brought enormous strides in health research. Ironically, epidemiology itself suffers from a major flaw: the end points that it correlates - largely, crude clinical symptoms - are at best surrogates of the underlying biological basis of the disease.

Most of what we call 'diseases' are a kaleidoscope of conditions, with distinct origins, prognoses, risk factors, genetic susceptibilities, and responses to therapy. Until now, epidemiology has of necessity investigated a disease as if it were 'one' disease, whereas many variants of it may respond differently to the factors under study - a major confounding variable. Moreover, even in the most intensively studied diseases, identified risk factors account for only a fraction of the variation in morbidity and mortality. Much remains to be discovered.

Research would be substantially more effective if it could better identify patients with subtypes of a disease, and acquire a better understanding of the underlying biological correlates. That is the lofty goal of a new European project to marry high-throughput post-genomic technologies and epidemiology in a syst ems biology approach dubbed 'genomic epidemiology' (see page 139).

Funding for the project is uncertain, and technological obstacles abound, but it deserves support. The scientists behind it are showing vision by thinking outside their disciplinary and institutional boxes. In marrying epidemiology and high-tech post-genomics, they may not only rejuvenate epidemiology, but also set a new standard for experimental design in biology. 